\title{
Determination of the Density of Energy States in a Quantizing Magnetic Field for Model Kane
}

\author{
G. Gulyamov, ${ }^{1}$ U. I. Erkaboev, ${ }^{2}$ and P. J. Baymatov ${ }^{3}$ \\ ${ }^{1}$ Namangan Engineering-Pedagogical Institute, 160103 Namangan, Uzbekistan \\ ${ }^{2}$ Physico-Technical Institute, NGO "Physics-Sun", Academy of Sciences of Uzbekistan, 100084 Tashkent, Uzbekistan \\ ${ }^{3}$ Namangan State University, 160119 Namangan, Uzbekistan
}

Correspondence should be addressed to U. I. Erkaboev; erkaboev1983@mail.ru

Received 22 July 2016; Accepted 14 September 2016

Academic Editor: Yuri Galperin

Copyright (C) 2016 G. Gulyamov et al. This is an open access article distributed under the Creative Commons Attribution License, which permits unrestricted use, distribution, and reproduction in any medium, provided the original work is properly cited.

For nonparabolic dispersion law determined by the density of the energy states in a quantizing magnetic field, the dependence of the density of energy states on temperature in quantizing magnetic fields is studied with the nonquadratic dispersion law. Experimental results obtained for $\mathrm{PbTe}$ were analyzed using the suggested model. The continuous spectrum of the energy density of states at low temperature is transformed into discrete Landau levels.

\section{Introduction}

In a study of the energy spectra of electrons in semiconductors, exceptional role was played by the application of quantum magnetic fields. As shown by Landau, in his classic work, the application of a magnetic field to a system of electrons causes a profound restructuring of the energy spectrum of electrons. It is accompanied by the appearance at certain values of the energy density of states singularities.

In the works of $[1,2]$, the temperature dependence of the density of states in quantizing magnetic fields was considered as the result of thermal broadening of the Landau levels. In those works studies showed that the density of states of the continuous spectrum measured at the temperature of liquid nitrogen at low temperatures turns into discrete Landau levels. Mathematical modeling of processes by using the experimental values of the density of states of the continuous spectrum makes it possible to calculate the discrete Landau levels. However, these works are considered only in the quadratic dispersion law. If the dispersion law is nonquadratic as, for example, electrons in the III-V compounds and II-VI, the effective mass is dependent on energy.

In the works of $[3,4]$, they determined the density of the energy states in a strong magnetic field at a temperature of liquid nitrogen. In these works the experimental results were compared with the model Kane in narrow-gap semiconductors. The influence of temperature on the density of the energy states in a quantizing magnetic field was not discussed.

The aim of this work is to determine the temperature dependence of the density of energy states in a quantizing magnetic field for the model Kane and the effect of temperature of a sample on the results of treatment of experimental data.

\section{Determination of the Density of Energy States in a Quantizing Magnetic Field for the Kane Dispersion Law}

In a magnetic field, the energy of free electrons with a quadratic dispersion law, and in view of the spinal level, splitting energy takes the following form $[5,6]$ :

$$
E\left(n, s, p_{z}\right)=\hbar \omega_{c}^{c}\left(n+\frac{1}{2}\right)+\frac{1}{2} s g \mu_{B} B+\frac{p_{z}^{2}}{2 m_{0}} .
$$

Here, we have $\omega_{c}^{c}$, the electron cyclotron frequency; $\mu_{B}$, Bohr magneton; $B$, the magnetic field induction; $s$, the spin quantum number, and $g$, a factor. 
In a magnetic field density of states for a parabolic band is determined by the following expression:

$$
\begin{aligned}
& N_{H}(E, H) \\
& =\frac{1}{4 \pi^{2}}\left(\frac{2 m^{*}}{\hbar^{2}}\right)^{3 / 2} \hbar \omega_{H} \sum_{n}\left\{E-\left(n+\frac{1}{2}\right) \hbar \omega_{H}\right\}^{-1 / 2} .
\end{aligned}
$$

However, if the energy dependence of the wave vector is not described by a quadratic form, such as for electrons in InSb energy levels of the charge carriers in the magnetic field are not equidistant, since cyclotron mass is determined by the expression

$$
m_{c}=\frac{\hbar^{2}}{2 \pi} \frac{\partial S}{\partial E}
$$

and therefore the cyclotron frequency depends on $E$ and $k_{z}$.

Nonparabolicity conduction band in compounds III-IV and II-VI is the result of interaction between the conduction and valence bands. In magnetic field energy levels for the three bands (apart from the heavy hole band that does not interact with them) are cubic equation [7]:

$$
\begin{aligned}
E_{N \pm} & \left(E_{N \pm}+E_{g}\right)\left(E_{N \pm}+E_{g}+\Delta\right) \\
& -P^{2}\left[k_{z}^{2}+(2 N+1) \frac{1}{L^{2}}\right]\left(E_{N \pm}+E_{g}+\frac{2}{3} \Delta\right) \\
& \pm \frac{P^{2} \Delta}{3 L^{2}}=0 .
\end{aligned}
$$

Here, $E_{N_{+}}$is energy electrons in the conduction band in view of spin in a quantizing magnetic field, $E_{g}$ is width bandgap, $\Delta$ is the spin-orbit splitting, and $P$ is the matrix element.

In our works, we consider narrow-gap semiconductors electrons that have a Kane dispersion law if the conditions $[8,9]$ are as follows:

$$
\left\{\begin{array}{c}
E(p)-E_{C} \ll E_{g}+\frac{2}{3} \Delta \\
\frac{2}{3} \Delta \gg E_{g}
\end{array}\right\} .
$$

From this condition of the cubic equation (4) reduced to the square, the solution of these electrons of the conduction band is given by

$$
\begin{aligned}
& E_{N \pm} \\
& =-\frac{E_{g}}{2} \\
& \quad+\frac{1}{2} \sqrt{E_{g}^{2}+4 E_{g}\left[\left(N+\frac{1}{2}\right) \hbar \omega_{c}+\frac{\hbar^{2} k_{z}^{2}}{2 m_{n}} \pm \frac{g_{0} \mu_{B} H}{2}\right]} .
\end{aligned}
$$

Equation (6) is applicable only for narrow-gap semiconductors.

We now find the number of states with energies in the interval between Landau levels.
We define the difference between the areas of the cross sections of the two surfaces of constant energy, the energy of which differs by

$$
\Delta S=\frac{2 \pi m_{c}}{\hbar^{2}} \Delta E=\frac{2 \pi m_{c}}{\hbar^{2}} \hbar \omega_{c}
$$

The number of states per unit area in the plane of $k_{x} k_{y}$ is quantized $\left(L_{x} L_{y} /(2 \pi)^{2}\right)$. Hence, the number of states between two quantum orbits can be written as follows:

$$
\frac{L_{x} L_{y}}{(2 \pi)^{2}} \Delta S=\frac{m \omega_{c}}{2 \pi \hbar} L_{x} L_{y} .
$$

From (6) we define $k_{z}$ without spin:

$$
k_{z}=\frac{(2 m)^{1 / 2}}{\hbar}\left(\frac{E^{2}+E_{g}\left(E-(N+1 / 2) \hbar \omega_{c}\right)}{E_{g}}\right)^{1 / 2} .
$$

We return now to the calculation of the density of states with a nonparabolic dispersion law in a magnetic field. The movement of free electrons along the $z$-axis is quantized by $k_{z}$. That is,

$$
k_{Z}=\frac{2 \pi}{L_{Z}} n_{Z}
$$

According to the expressions (9) and (10) the number of states of the energy interval from $(N+1 / 2) \hbar \omega_{c}$ to $E$ is

$$
n_{Z}=\frac{(2 m)^{1 / 2}}{\pi \hbar} \cdot\left(\frac{E^{2}+E_{g}\left(E-(N+1 / 2) \hbar \omega_{c}\right)}{E_{g}}\right)^{1 / 2} .
$$

The total number of bulk quantum states with energies less than $E$, as well, is

$$
\begin{aligned}
N(E)= & \frac{L_{x} L_{y} L_{z} m^{3 / 2}}{\pi^{2} \hbar^{3}} \hbar \omega_{c} \\
& \cdot \sum_{N=0}^{N_{\max }}\left(\frac{E^{2}+E_{g}\left(E-(N+1 / 2) \hbar \omega_{c}\right)}{E_{g}}\right)^{1 / 2} .
\end{aligned}
$$

As a result, we define the energy density of states per unit volume excluding spin Kane dispersion law in a magnetic field:

$$
\begin{gathered}
N_{S}^{n}(E, H)=\frac{d N(E)}{d E}=\frac{(m)^{3 / 2}}{(2)^{1 / 2} \pi^{2} \hbar^{3}} \frac{\hbar \omega_{c}}{2} \\
\cdot \sum_{N=0}^{N_{\max }} \frac{\left(2 E / E_{g}\right)+1}{\sqrt{\left(E^{2} / E_{g}\right)+E-(N+1 / 2) \hbar \omega_{c}}} .
\end{gathered}
$$

Here, $N_{S}^{n}(E, H)$ is the density of the energy states of a Kane dispersion law in a quantizing magnetic field.

At $E_{g} \rightarrow \infty$ expression (13) goes into a parabolic dispersion law (2). In this expression, the temperature-induced broadening of the energy levels is disregarded. 


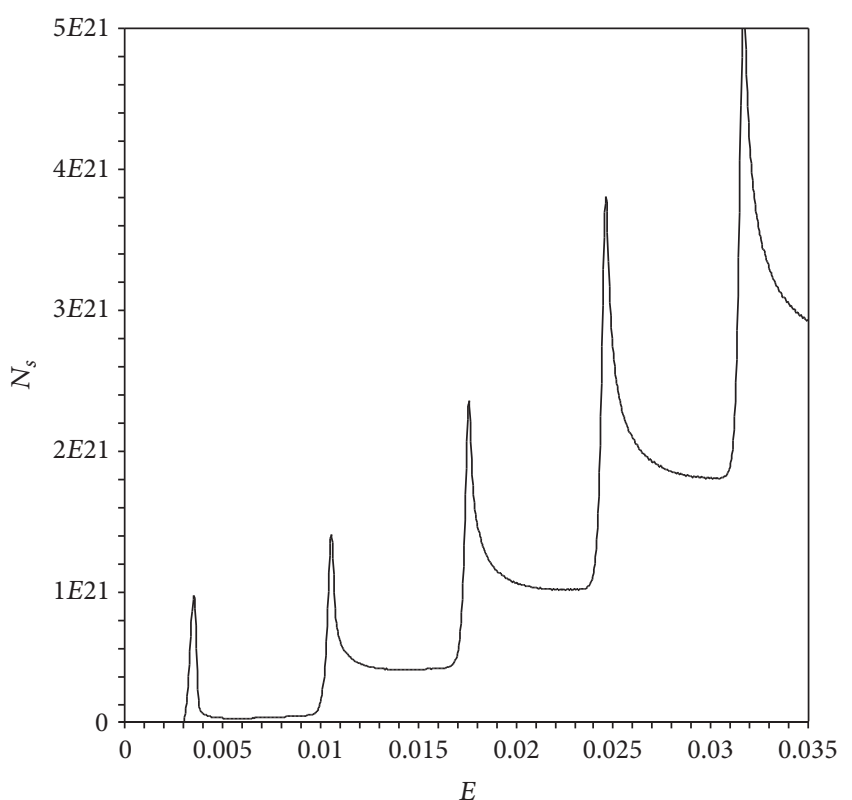

FIGURE 1: Density of energy states in a high magnetic field $B=2 \mathrm{~T}$ at a temperature $T=1 \mathrm{~K}$ as calculated using formula (14).

\section{The Influence of Temperature on the Density of States with Energy Kane Dispersion Law in a Strong Magnetic Field}

In the work of [10], the determination of the thermodynamic density of states in a strong magnetic field was discussed. The thermodynamic density of states of such a system is a set of delta function peaks separated from each other on $\hbar \omega_{c}$.

Thermal broadening of the levels in a magnetic field gives rise to the smoothing of discrete levels. Thermal broadening is to be taken into account using a derivative of the energy distribution function of the Fermi-Dirac $d f_{0}(E, \mu, T) / d E$. At absolute zero of temperature, the $d f_{0}(E, \mu, T) / d E$ function transforms into the delta function of Dirac.

In order to take into account the temperature dependence of the density of states, we expend $N_{S}^{n}(E, H)$ into series with respect to $d f_{0}(E, \mu, T) / d E$ functions. We then obtain the density of energy states depending on temperature [1]:

$$
N_{S}^{n}(E, H, T)=\sum_{i=1}^{n} N_{H}^{n}\left(E_{i}, H\right) \frac{d f_{0}\left(E_{i}, \mu, T\right)}{d E} .
$$

Here, $H$ is the magnetic field strength, $\omega_{c}=e H / m^{*} c$ is the cyclotron frequency, $m^{*}$ is the cyclotron effective mass, and $N_{H}^{n}\left(E_{i}, H\right)$ is the density of states in a quantizing magnetic field at the absolute zero of temperature.

The corresponding expression at $T \rightarrow 0$ transforms into (13). In this case, the Landau levels are manifested sharply. In this case, the Landau levels manifest themselves more distinctly. As the temperature is elevated, sharp increases in the spectrum start to be smoothed (Figure 1) and, at $k T \sim$ $\hbar \omega$, oscillations in the density of states gradually disappear at relatively high temperatures $k T>\hbar \omega$, and $N_{S}^{n}(E, H, T)$ transforms into the continuous density of states and will not

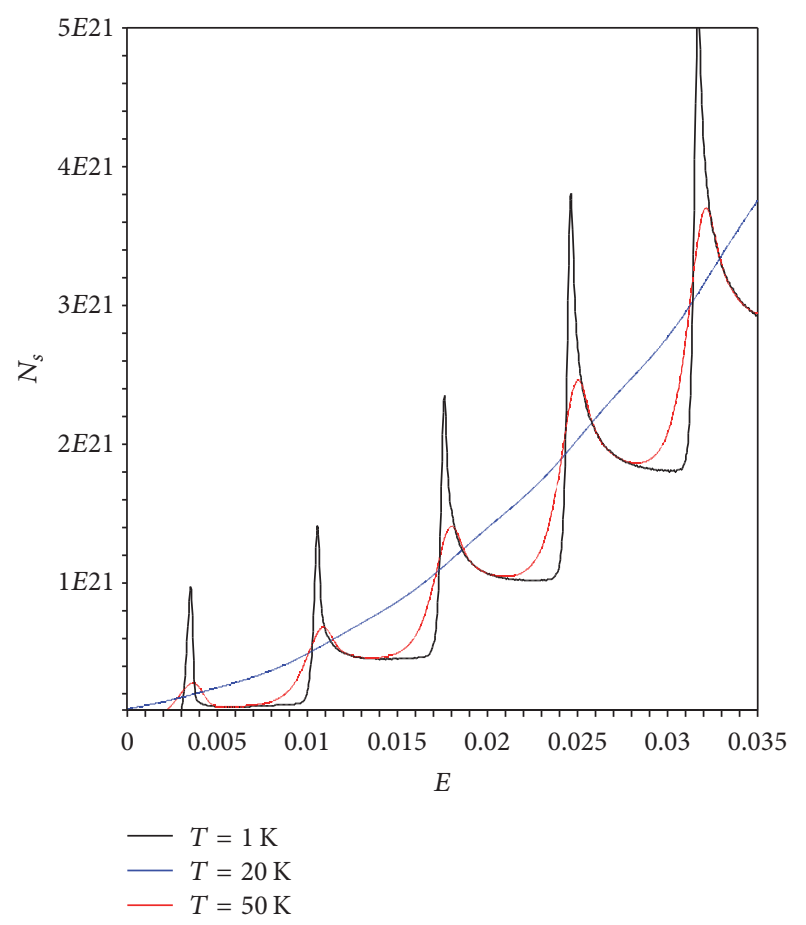

FIGURE 2: Temperature dependence of the density of energy states in a quantizing magnetic field is calculated using a model Kane.

be sensitive to a magnetic field. The quantity $N_{S}^{n}(E, H, T)$ is transformed into the density of states in the absence of a magnetic field. This makes it possible to obtain the density of states, which depends on temperature. In Figure 1, we show the density of states in a magnetic field for InSb for the following parameters [7]:

$$
\begin{aligned}
m_{n}^{*} & =\frac{1}{70} m_{0}, \\
E_{g}(0) & =0,234 \mathrm{eV}, \\
B & =2 T \\
\text { or } \hbar \omega_{c} & \approx 1,5 \cdot 10^{-3} \mathrm{eV} .
\end{aligned}
$$

At the temperature of $T=1 \mathrm{~K}, k T=8,6$. $10^{-5} \mathrm{eV}\left(\hbar \omega_{c} / k T\right)=5,7 \cdot 10^{2}, k T \ll \hbar \omega_{c}$. At such low temperatures, the effect of thermal broadening is slight and the density of states is not affected by the deviation of $N_{S}^{n}(E, H, T)$ from the ideal shape, which is supposedly unaffected by temperature. In Figure 2, we show the function $N_{S}^{n}(E, H, T)$ for temperatures 1,20 , and $50 \mathrm{~K}$. As can be seen from Figure 2, as temperature is increased, the sharp peaks corresponding to Landau levels related to the quantization of electron energy levels in the plane perpendicular to the magnetic field are gradually smoothed. This brings about the fact that, at a temperature of $T=20 \mathrm{~K}, k T \approx \hbar \omega_{c}$, the peaks of the Landau levels become insignificant. At a temperature of $50 \mathrm{~K}$, the peaks of the Landau levels become practically indistinguishable and coincide with the density of states in the absence of a quantizing magnetic field. 


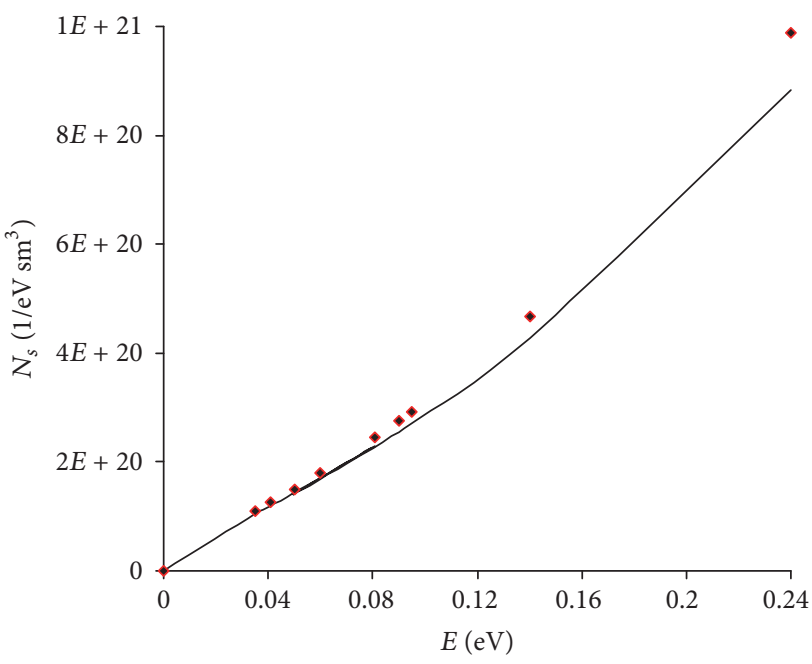

a)

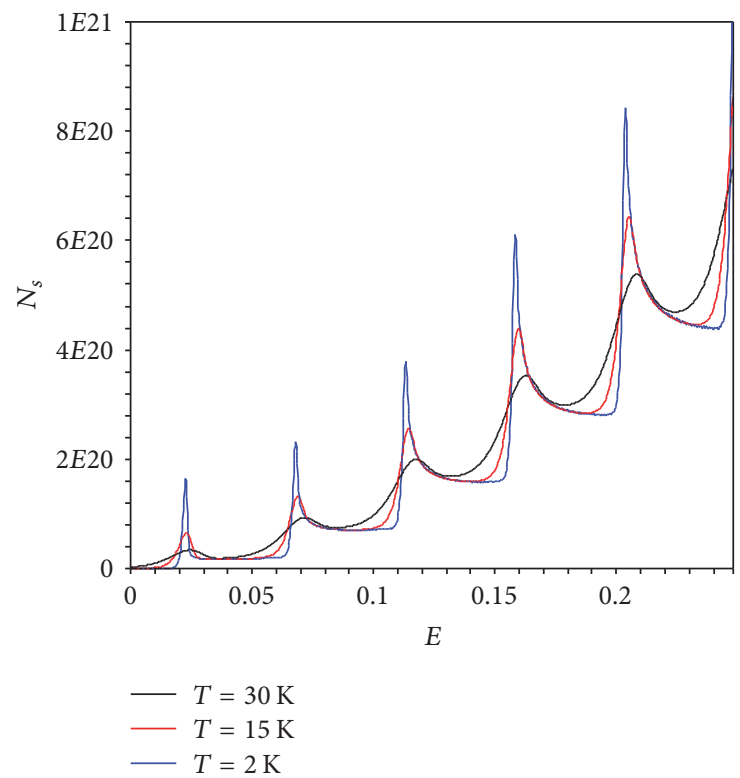

(b)

FIGURE 3: Determination of the change in the density of energy states with decreasing temperature in high magnetic fields using the Kane model. (a) The experimental data (dots) for PbTe at $H=30 \mathrm{kOe}[3,4]$ and the theory of calculation (solid line) for $T=77 \mathrm{~K}$. (b) The density of the energy states of a nonparabolic dispersion law for three different values of the temperature in a magnetic field.

\section{Comparison of Theory and Experiment}

Let us consider the energy density of states of narrow-gap semiconductors in a quantizing magnetic field. Figure 3(a) shows the density of states at the temperature of measurements $T=77 \mathrm{~K}$ and $H=30 \mathrm{kOe}(B=3 T) \mathrm{PbTe}$ $[3,4]$. Within experimental accuracy, the density of states is continuous and steadily increasing the smooth function of energy. In this figure, the oscillations of the density of states are not observed. This indicates that $\hbar \omega_{c}<k T$. Let us expand this representation of the density of states in series with respect to $d f_{0}(E, \mu, T) / d E$ functions. In the expansion, the initial term should be represented by the ideal $N_{S}^{n}(E, H, T)$ from formula (13) (Figure 3(a)). We will position the $N_{S}^{n}(E, H, T)$ peaks according to the values of the energy multiplied by $\hbar \omega_{c}$; however, we consider the height of peaks depending on energy. Then, choosing the coefficients in the sum before $N_{S}^{n}(E, H, T)$, we fit the theoretical values of $N_{s}(E, H)$ to experimental ones with the adjustable parameter being exactly the coefficient at $N_{s}\left(E_{0}, H\right)$. Further on, we will lower the temperature in formula (14). Starting with a temperature close to $20 \mathrm{~K}$, the smooth curve starts to oscillate and the Landau levels become distinct (Figure 3(b)). Thus, at a temperature corresponding to $k T \ll \hbar \omega_{c}$, the Landau levels start to distinctly stand out as separate peaks of $N_{S}^{n}(E, H, T)$.

\section{Conclusion}

We developed a new method for determining the density of the energy states in a quantizing magnetic field for the Kane model. For a nonquadratic dispersion law, it was shown that the density of states in a strong magnetic field at an increased temperature coincides with the density of states in the sample without a magnetic field. It is shown that the Landau levels broaden due to the thermal effect as temperature is increased and $N_{s}$ is transformed into the density of states in the absence of a magnetic field. With the help of suggested model, we studied the experimental results for $\mathrm{PbTe}$ [3]. On the basis of this model, we used data on the high temperature density of states to calculate the low temperature density of states. Simulation of the temperature dependence is possible to determine the Landau levels in PbTe in broad temperature spectra.

\section{Competing Interests}

The authors declare that they have no competing interests.

\section{References}

[1] G. Gulyamov, U. I. Erkaboev, and N. Yu. Sharibaev, "Effect of temperature on the thermodynamic density of states in a quantizing magnetic field," Semiconductors, vol. 48, no. 10, pp. 1287-1292, 2014.

[2] G. Gulyamov, U. I. Erkaboev, and N. Yu. Sharibaev, "Simulation of the temperature dependence of the density of states in a strong magnetic field," Journal of Modern Physics, vol. 5, no. 8, pp. 680-685, 2014.

[3] Yu. I. Ravich, V. A. Efimova, and I. A. Smirnov, Investigation Methods of Semiconductors in Application to Plumbum Halcogenids PbTe, PbSe and PbS, Nauka, Moscow, Russia, 1968 (Russian).

[4] I. N. Dubrovsky and Y. I. Ravich, "Study parabolic conduction band of $\mathrm{PbTe}$ by thermo power measurements in a strong magnetic field," Physics of the Solid State, vol. 8, no. 5, pp. 14551460, 1996. 
[5] N. B. Brandt and V. A. Kulbachinsky, Quasiparticles in Condensed Matter Physics, chapter 6, Fizmatlit, Moscow, Russia, 2007 (Russian).

[6] J. Ziman, The Principles of the Theory of Solids, chapter 9, Mir, Moscow, Russia, 1974.

[7] I. M. Tsidilkovsky, Electrons and Holes in Semiconductors, Nauka, Moscow, Russia, 1972 (Russian).

[8] I. S. Dubitskiy and A. M. Yafyasov, "On the field effect in thin films of semiconductors with Kane's charge-carrier dispersion relation," Semiconductors, vol. 48, no. 3, pp. 312-319, 2014.

[9] N. V. Pavlov and G. G. Zegrya, "Effect of nonparabolicity of the electron and light-hole energy spectrum on the optical properties of heterostructures with deep $\mathrm{AlSb} / \mathrm{InAs}_{0.86} \mathrm{Sb}_{0.14} / \mathrm{AlSb}$ quantum wells," Semiconductors, vol. 49, no. 5, pp. 604-614, 2015.

[10] I. V. Kukushkin, S. V. Meshkov, and V. B. Timofeev, "Twodimensional electron density of states in a transverse magnetic field," Soviet Physics Uspekhi, vol. 31, no. 6, pp. 511-534, 1988. 

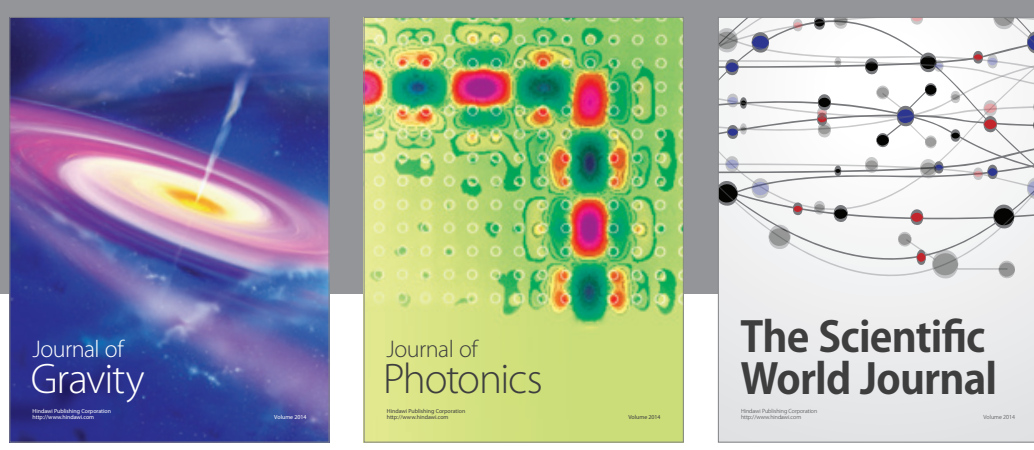

The Scientific World Journal
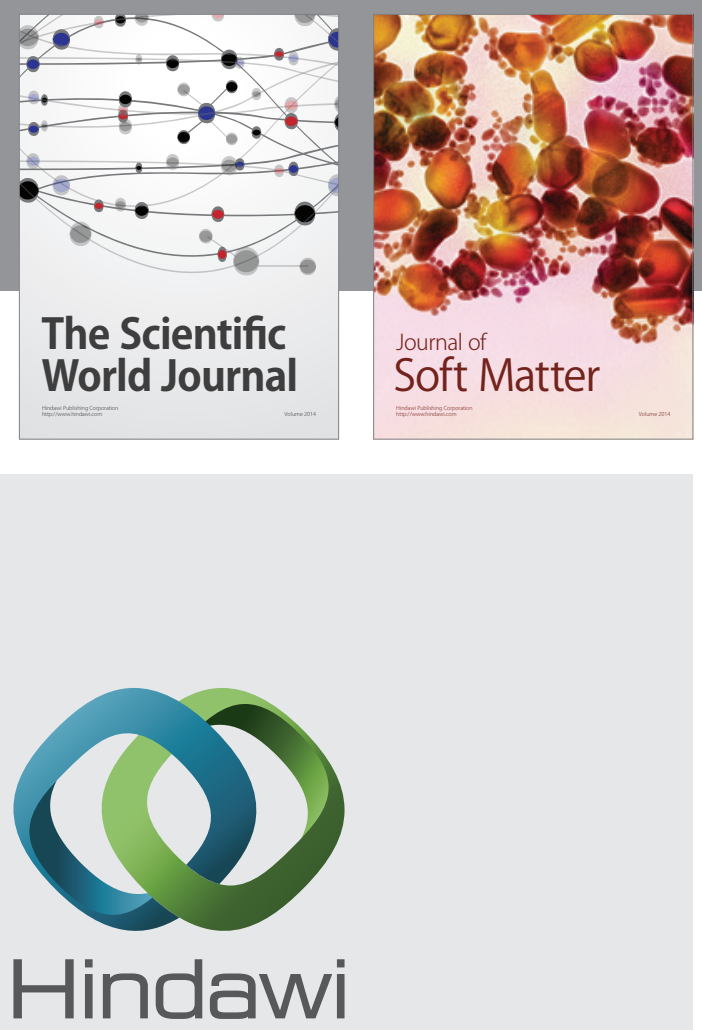

Submit your manuscripts at

http://www.hindawi.com

nternational Journal of

Statistical Mechanics
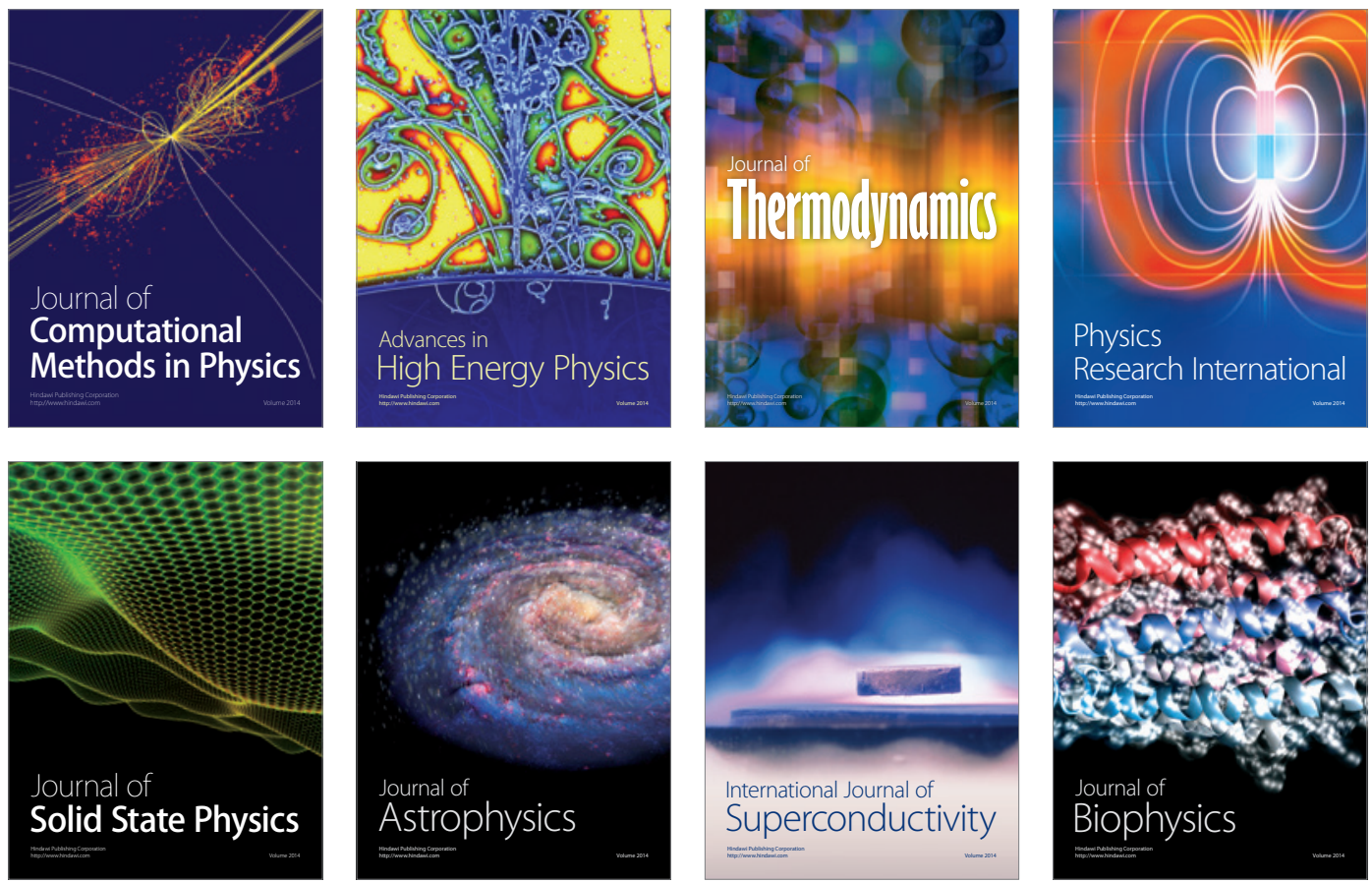
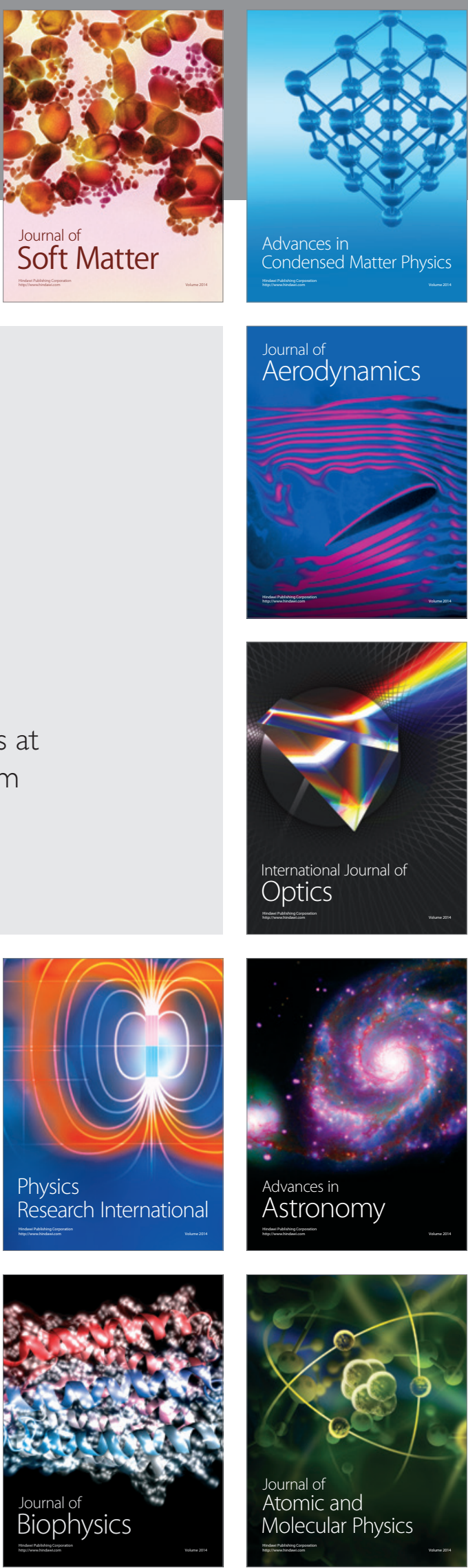